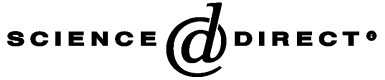

Radiation Physics and Chemistry 68 (2003) 363-368
Radiation Physics

and

Chemistry

www.elsevier.com/locate/radphyschem

\title{
Positron and positronium in porous media: zeolites
}

\author{
Zs. Kajcsos ${ }^{\mathrm{a}, \mathrm{b}, *}$, L. Liszkay ${ }^{\mathrm{a}, 1}$, G. Duplâtre ${ }^{\mathrm{c}}$, L. Lohonyai $^{\mathrm{a}}$, L. Varga ${ }^{\mathrm{a}}$, K. Lázár ${ }^{\mathrm{d}}$, \\ G. Pál-Borbély ${ }^{\mathrm{e}}$, H.K. Beyer ${ }^{\mathrm{e}}$, P. Caullet ${ }^{\mathrm{f}}$, J. Patarin ${ }^{\mathrm{f}}$, A.P. de Lima ${ }^{\mathrm{b}}$, \\ C. Lopes Gil ${ }^{\mathrm{b}}$, P.M. Gordo ${ }^{\mathrm{b}}$, M.F. Ferreira Marques ${ }^{\mathrm{b}, \mathrm{g}}$ \\ ${ }^{\text {a }}$ KFKI Research Institute for Particle and Nuclear Physics, P.O. Box 49, Budapest H-1525, Hungary \\ ${ }^{\mathrm{b}}$ Departamento de Física, University of Coimbra, Coimbra 3004-516, Portugal \\ ${ }^{\mathrm{c}}$ Laboratoire de Chimie Nucléaire, IReS, IN2P3/CNRS, B.P. 28, 67037 Strasbourg, Cedex 2, France \\ ${ }^{\mathrm{d}}$ Institute of Isotope and Surface Chemistry, P.O. Box 77, Budapest H-1525, Hungary \\ ${ }^{\mathrm{e}}$ Central Research Institute for Chemistry, P.O. Box 19, Budapest H-1525, Hungary \\ ${ }^{\mathrm{f}}$ Laboratoire de Matériaux Minraux, URA CNRS 428 3, rue A. Werner 68093 Mulhouse, Cedex, France \\ ${ }^{\mathrm{g}}$ Instituto Superior de Engenharia, Coimbra 3031-601, Portugal
}

\begin{abstract}
Correlations between positronium (Ps) production features and positron annihilation (PA) parameters were investigated in various zeolite samples (MFI, mordenite, Na-Y). The contribution of $3 \gamma$-annihilation on data registration was closely examined. Long lifetimes up to $135 \mathrm{~ns}$ and triplet Ps (o-Ps) fractions up to $30 \%$ were found. Even low amounts of adsorbed gases or water drastically change the PA pattern. It is concluded that in addition to the crystallographic free sites, large structural discontinuities also play an important role as they can trap Ps and provide it with vacuum-like survival conditions.
\end{abstract}

(C) 2003 Elsevier Science Ltd. All rights reserved.

Keywords: Zeolites; Long-living positronium; Porous structure; $3 \gamma$-annihilation

\section{Introduction}

Positron annihilation (PA) studies in microcrystalline and porous media reveal a great variety of annihilation modes, with a substantial contribution from long-living ortho-positronium (o-Ps) states as was discovered long ago (Paulin and Ambrosino, 1966). In the decades following this finding, growing attention has been paid to zeolites (e.g., Debowska et al., 1988; Ito et al., 1988; Wang and Jean, 1988; Hasegawa et al., 1992; Chen et al., 1995) which, among those materials, possess features

\footnotetext{
*Corresponding author. KFKI Research Institute for Particle and Nuclear Physics, P.O. Box 49, Budapest H-1525, Hungary. Fax: + 36-1-395-9151.

E-mail address: kajcsos@rmki.kfki.hu (Z. Kajcsos).

${ }^{1}$ Present address: Institut für Nukleare Festkörperforschung, Universität der Bundeswehr, Werner-Heisenberg Weg 39, Neubiberg DE-85577, Germany.
}

that are highly attractive to technological applications. However, PA measurements yielded in some cases widely differing results for nominally the same samples, pointing to the role of yet unclear factors. There may appear up to five different lifetimes, from about 0.15 up to $140 \mathrm{~ns}$, with intensities of the longest components as high as 30-40\% (Kajcsos et al., 2001; Wang et al., 2001). Those long-living states decay dominantly by $3 \gamma$ annihilation, and their existence testifies that $o$-Ps virtually does not interact with its surroundings.

Notwithstanding the efforts in the last few years, there are still open questions with respect to the mechanism governing the fate of positrons and the formation and interactions of positronium (ps) in a solid structure with abundant free volume. The simple quantum-well model (Tao, 1972; Eldrup et al., 1981) correlating the $o$-Ps lifetime to the size of those voids where it decays, has been further developed recently (e.g., Goworek et al., 2000) by taking into account non-spherical geometry as 
well. However, in some cases, long lifetime values, much exceeding the values corresponding to the known crystallographic free volumes were found.

Correlation of the PA parameters with, e.g., the cage size, the presence of adsorbed water, etc., was sought and has been established in some cases, but there is a substantial discrepancy among the values in the literature. It has been suggested (Kajcsos et al., 2001) that in addition to the sample synthesis and treatment, which both have an influence, possible changes in the $3 \gamma /$ $2 \gamma$ ratio (due to e.g., interactions of $o$-Ps with any additives present-surface-bound ions, gases, etc.,shortening the $o$-Ps lifetime and thus increasing the probability of annihilation via $2 \gamma$-annihilation) may also strongly influence the PA parameters. The positron lifetime spectrometers routinely employed are not optimised for recording the $3 \gamma$-annihilation decay with high efficiency and without distortion; most generally, only scattered attempts have been made to correct those influences (Dauwe, 1992). In the present work, it was our goal to further study the influence of sample treatment (including the investigation of water removal) and the above-mentioned effects of non-linear recording conditions, connecting our efforts to the previous systematic studies started both on silicalite-1 and also on the $\mathrm{X}$-, Y-zeolites and on mordenite, with wellknown high technological value.

\section{Experimental}

Special care has been taken to select well-characterised samples, known to offer high Ps yield (Duplâtre et al., 1996; Varga et al., 1996). The MFI silicalite-1 and mordenite samples were produced in Mulhouse in accordance with the technological processes described earlier (Guth et al., 1986), the $\mathrm{Na}-\mathrm{Y}$ samples were purchased commercially (Grace). The grain size of the samples varied form about $30 \times 10 \times 10 \mu \mathrm{m}^{3}$ for the silicalite-1 down to $1-5 \mu \mathrm{m}$ grain size diameter for the $\mathrm{Na}-\mathrm{Y}$. Besides thermogravimetry ( $\mathrm{Tg}$ ) measurements, the structure characterisation of the samples was performed using X-ray diffractiometry and NMR spectrometry.

The PA experiments included lifetime spectroscopy (LT), Doppler broadening technique, the recording of the full energy distribution (FED) of the annihilation radiation and the extended Doppler broadening (XDB) technique.

All PA experiments were performed on samples prepared by pressing $(0.3 \mathrm{GPa})$ the zeolite powder into disks of $8-13 \mathrm{~mm}$ diameter and $1-1.5 \mathrm{~mm}$ thickness. The ${ }^{22} \mathrm{Na}$ source $(0.5-2.0 \mathrm{MBq}$ activity, held between thin Kapton or Ni foils) was sandwiched between two sample disks. The temperature-dependent water removal from the mordenite and $\mathrm{Na}-\mathrm{Y}$-zeolite was studied in the
90-430 K region by LT, FED and XDB measurements performed on samples placed in an evacuated sampleholder cryostat-oven, with temperatures controlled to $1 \mathrm{~K}$ accuracy. The weight of the samples was measured before and after water removal.

The LT spectrometer, featuring XP 2020Q photomultipliers equipped with $\mathrm{NE} 111$ and $\mathrm{BaF}_{2}$ scintillators, on the start and stop lines, respectively, consists of a fast-fast coincidence system which was tuned for highefficiency recording of the $3 \gamma$-events employing wide energy selection windows $(50 \%$ on the start and $80 \%$ on the stop lines), giving time resolution values for the ${ }^{60} \mathrm{Co}$ prompt spectrum of $420 \mathrm{ps}$ in FWHM. The LT spectra were recorded in $\mathrm{PC}$-based $4 \mathrm{k}$ MCAs with a time range of $2 \mu \mathrm{s}$, and integral counts of $2 \times 10^{6}-1 \times 10^{7}$. Evaluation of the LT spectra was carried out by using a fitting program (based on the MINUIT subroutine package) purpose-tailored to achieve fast convergence also in case of lifetimes stretching over a dynamic span of 1:1000. Appropriate source corrections were taken into account. The intensities of the long-lived components are also subject to corrections due to two main reasons: the spectra are distorted by the unequal efficiencies for the detection of $2 \gamma$-and $3 \gamma$-events, and the registered spectra mirror the "survival proportions" of the various components and not those of the "birth fractions". Corrections for the first effect require considerations (Duplâtre et al., 2003) which exceed the framework of the present article (we note here only that in our case the $\rho$ factor is 1.79 , meaning that the $3 \gamma$-events are overaccentuated by this factor) but corrections for the second kind of distortion can be performed more easily (Kajcsos et al., 2001; Liszkay et al., 2001). The $3 \gamma$ fraction, $f_{3 \gamma}$, can be expressed as follows: $f_{3 \gamma}=I{ }^{*}$ $\tau_{\mathrm{L}} / \tau_{o \text {-Ps }}$, where $\tau_{\mathrm{L}}$ is the long lifetime reduced from the value of $\tau_{o \text {-Ps }}=142 \mathrm{~ns}$ (the $o$-Ps lifetime in vacuum) by the interaction of $o$-Ps with the surrounding matter, and $I^{*}{ }_{\mathrm{L}}$ is the real physical "birth population" intensity of the long-lived component. The value of $f_{3 \gamma}$ may be slightly distorted by the overlap of the $3 \gamma$-energy spectrum with the $0.511 \mathrm{MeV}$ annihilation peak (AP) and by changes in the $\gamma$-absorption. $I^{*} \mathrm{~L}$ can be calculated from the measured $\tau_{\mathrm{L}}$ and $f_{3 \gamma}$ which can be determined independently, e.g., from FED or XDB measurements.

The FED spectra were recorded in the $40-1350 \mathrm{keV}$ range with a Canberra HPGe detector (24\% efficiency and $1.08 \mathrm{keV}$ resolution at $0.5 \mathrm{MeV})$, coupled to a Microfast $16 \mathrm{k}$ PC MCA. The XDB spectra were selected out of the FED data sets applying a region of interest setting of $450-550 \mathrm{keV}$.

For the characterisation of the $3 \gamma$-contribution to the FED or XDB spectra, one may consider several options. Changes in the relative integral counts $A_{2} \gamma=$ $A_{0.51 \mathrm{MeV}} / A_{1.28 \mathrm{MeV}}$ in the $511 \mathrm{keV}$ AP are proportional to the amount of positrons leaving the $2 \gamma$-annihilation 
channel and annihilating via $3 \gamma$-decay (Liszkay et al., 2001). As a reference for the "pure" $2 \gamma$ annihilation case, the spectra measured on water saturated samples were used (the negligible $3 \gamma$-annihilation was proven by lifetime measurements: no lifetimes above $2 \mathrm{~ns}$ were found). The relative number of positrons annihilating through the $3 \gamma$-channel, i.e., the $3 \gamma$-fraction $\left(f_{3 \gamma}\right)$ can be expressed as $f_{3 \gamma}=1-A_{2 \gamma} / A_{2 \gamma}^{0}$, where $A_{2 \gamma}^{0}$ is the "pure" $2 \gamma$-reference. To a first approximation, the contribution from the $3 \gamma$-annihilation to the $511 \mathrm{keV}$ peak can be generally neglected. A similar characterisation can be performed (Kajcsos et al., 1995) by considering the left and right sections of the $511 \mathrm{keV}$ AP: the $\mathrm{L} / \mathrm{R}$ values (left/right plateau regions of AP) give information on the $3 \gamma$-radiation emitted by the annihilation of $o$-Ps, the main difference to the consideration of the changes in AP being only the lower statistical accuracy. The $L / R$ values had to be scaled as well.

A good reproducibility of the PA results was found by recording spectra over several days, then completely reprocessing the samples and repeating the experiments.

$\mathrm{Tg}$ was carried out in a dry nitrogen flow with $0.5 \mathrm{~K} /$ min heating rate, which was kept constant at this value during the positron measurements as well.

\section{Results and discussion}

Previous experiments (Kajcsos et al., 1997) have shown that in most cases, a satisfactory fit of the LT spectra requires at least four lifetime component groups: $0.2-0.5 \mathrm{~ns}$, summing up the $p$-Ps and $\mathrm{e}^{+}$-annihilations, $1-5 \mathrm{~ns}$ for $o$-Ps trapped in (crystallographic) microvoids of the lattice, $10-90 \mathrm{~ns}$ for $o$-Ps decaying in larger spaces and $100-135 \mathrm{~ns}$ for $o$-Ps decaying, at least in part, outside of the crystallites, in the intergrain vacuum space.

Recently, on the basis of measurements of capillary gas condensation, it was recognised (Duplâtre et al., 2003) that a five-component fit gives physically reliable results, resolving the $1-5 \mathrm{~ns}$ component into two subcomponents, so this assumption was also used here. The LT results for a MFI silicalite-1 sample, which was produced so as to contain "structural defects" (Chezeau et al., 1991) are shown in Table 1. Making use of the simultaneous XDB measurements and also employing our correction method (Duplâtre et al., 2003) for the differing efficiency of the registration of $3 \gamma$ - and $2 \gamma$ events, respectively, the intensity values, corrected back to the "birth population" fractions are given in Table 2.

Due to the comparatively high time definition used, the absolute values of the shortest lifetimes cannot be regarded as similarly reliable as the longer ones. With respect to the longer lifetime values found for the MFI sample, quantitatively only $\tau_{2}$ and $\tau_{3}$ could be correlated on the basis of the simple quantum-well model with
Table 1

Lifetimes and (uncorrected) intensities of the $o$-Ps states in evacuated MFI silicalite-1, at $294 \mathrm{~K}$

\begin{tabular}{llllllll}
\hline$\tau_{2}(\mathrm{~ns})$ & $\tau_{3}(\mathrm{~ns})$ & $\tau_{4}(\mathrm{~ns})$ & $\tau_{5}(\mathrm{~ns})$ & $I_{2}(\%)$ & $I_{3}(\%)$ & $I_{4}(\%)$ & $I_{5}(\%)$ \\
\hline 2.05 & 5.6 & 35.1 & 135.4 & 24.1 & 2.6 & 2.5 & 28.1 \\
\hline
\end{tabular}

The errors are \pm 0.5 abs. $\%$.

Table 2

Measured $\left(I_{i}\right)$ and true $I_{i}^{t}$ lifetime intensities of the $o$-Ps components in MFI silicalite- 1 at $294 \mathrm{~K}$

\begin{tabular}{lllll}
\hline & $I_{2}(\%)$ & $I_{3}(\%)$ & $I_{4}(\%)$ & $I_{5}(\%)$ \\
\hline Raw intensity & 24.1 & 2.6 & 2.5 & 28.1 \\
Corrected intensity & 27.2 & 2.9 & 2.4 & 18.5 \\
\hline
\end{tabular}

$I_{i}^{t}$ values are corrected due to the shortening of the lifetimes and also due to the overall sensitivity difference for the $2 \gamma / 3 \gamma$ detection, expressed by the factor $\rho$, in our case $\rho=1.79$. The errors are $\pm 0.2 \%$ with respect to the absolute values.

crystalline free spaces (excluded volumes, intersections of channels, or the channels themselves, etc.,); no crystallographic spaces are present which could account for the very long lifetime values found for $\tau_{4}$ and $\tau_{5}$. Consequently, those possibly correspond to $o$-Ps annihilating from defected sites of the lattice, the so-called mesopores. The $o$-Ps trapped therein due to the inertness of the wall experiences vacuum-like conditions and survives appropriately long. This assumption is supported also by the PA results on capillary gas condensation and by measurements performed on zeolites immersed into liquids with small or big molecules (Kajcsos et al., 2001).

Water removal studies on the mordenite and $\mathrm{Na}-\mathrm{Y}$ zeolite samples (some typical data are listed in Table 3) have shown that both samples lose a high amount of water. The FED/XDB measurements (Fig. 1) clearly show that Ps formation starts already at the onset of water removal, as indicated by the large increase in the $S$-parameter, denoting abundant $p$-Ps formation. A high $o$-Ps yield, however, becomes apparent only at a much higher degree of water removal, as also proven by simultaneous LT and FED measurements (Fig. 2). The correlation of the $o$-Ps abundance with the water content is also shown by the monotonous decrease of $f_{3 \gamma}$ when the dry, evacuated sample is exposed to air and some water vapour is being adsorbed. Nonetheless, the total Ps yield seems to remain constant, the accompanying increase in the $S$-parameter possibly showing an $o$-Ps/ $p$-Ps conversion. For a repeated cooling-heating cycle (with no introduction of water), the level of the Ps production is maintained in the temperature range down to $150 \mathrm{~K}$.

Considering the growing contribution of $3 \gamma$-events upon evacuation with increasing temperature, this finding offers the means to establish the specific 
Table 3

Lifetime data for the long-lived $o$-Ps component of zeolite samples

\begin{tabular}{lcclll}
\hline Sample & \multicolumn{2}{c}{ Before heat treatment } & & \multicolumn{2}{c}{ After heat treatment } \\
\cline { 2 - 3 } \cline { 5 - 6 } & $\tau_{\mathrm{L}}(\mathrm{ns})$ & $I_{\text {long }}(\%)$ & & $\tau_{\mathrm{L}}(\mathrm{ns})$ & $I_{\text {long }}(\%)$ \\
\hline $\mathrm{Na}-\mathrm{Y}$ & $105.5(3)$ & $1.0(1)$ & & $109.5(3)$ & $16.8(1.5)$ \\
Mordenite & $55(1.5)$ & $2.0(1)$ & & $97(3)$ & $15(1.5)$ \\
\hline
\end{tabular}

$I_{\text {long }}$ represents the percentage of $o$-Ps that contributes to $\tau_{\mathrm{L}}$ (corrected using the FED/XDB data as well).

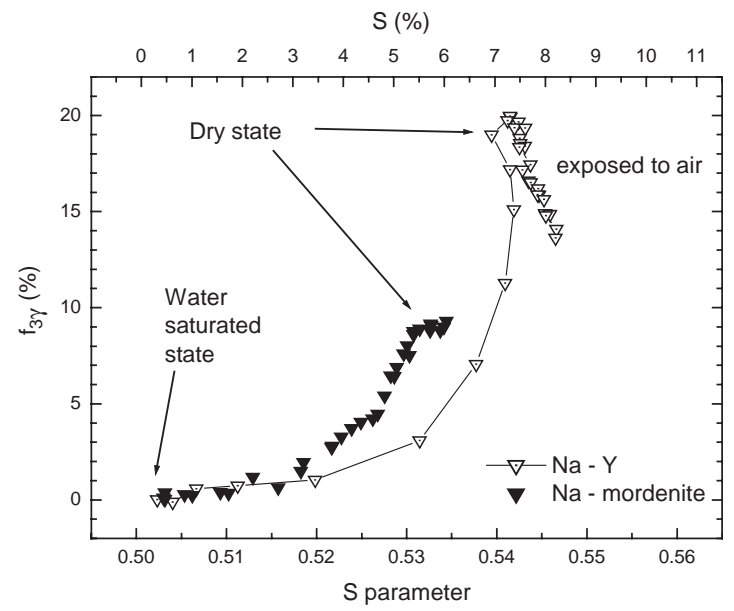

Fig. 1. The $S-f_{3 \gamma}$ plot ( $S$-parameter vs. $3 \gamma$-fraction) displaying the evolution of the Ps-formation during the vacuum heating water removal in mordenite and in $\mathrm{Na}-\mathrm{Y}$. Increase in the $3 \gamma$ ratio indicates high $o$-Ps formation. The temperature cycling was the same for all samples: a heating up from room temperature to $430 \mathrm{~K}$ has been followed by a cooling down to $150 \mathrm{~K}$. The $\mathrm{Na}-\mathrm{Y}$ sample was exposed to air after water removal, leading to a subsequent decrease of the $f_{3 \gamma}$ and an increase in the $S$-value.

energy-dependent contribution of this annihilation mode to the FED spectra. Normalising the $1.28 \mathrm{MeV}$ peaks to the same area, the difference among the FED spectra referring to "wet" and "dry" state of samples has been built (Fig. 3). The $511 \mathrm{keV}$ peak is not shown here as the difference in this peak has just the opposite sign and would disturb well-visible scaling. It is to be mentioned here that those differences could be best scaled by applying a strong magnetic field, affecting a saturation level magnetic quenching of $o$-Ps (Duplâtre et al., 1996).

\section{Conclusions}

The results reported here clearly draw attention to the strong influence of the sample treatment, and also indicate the difficulty of full removal of adsorbed water.

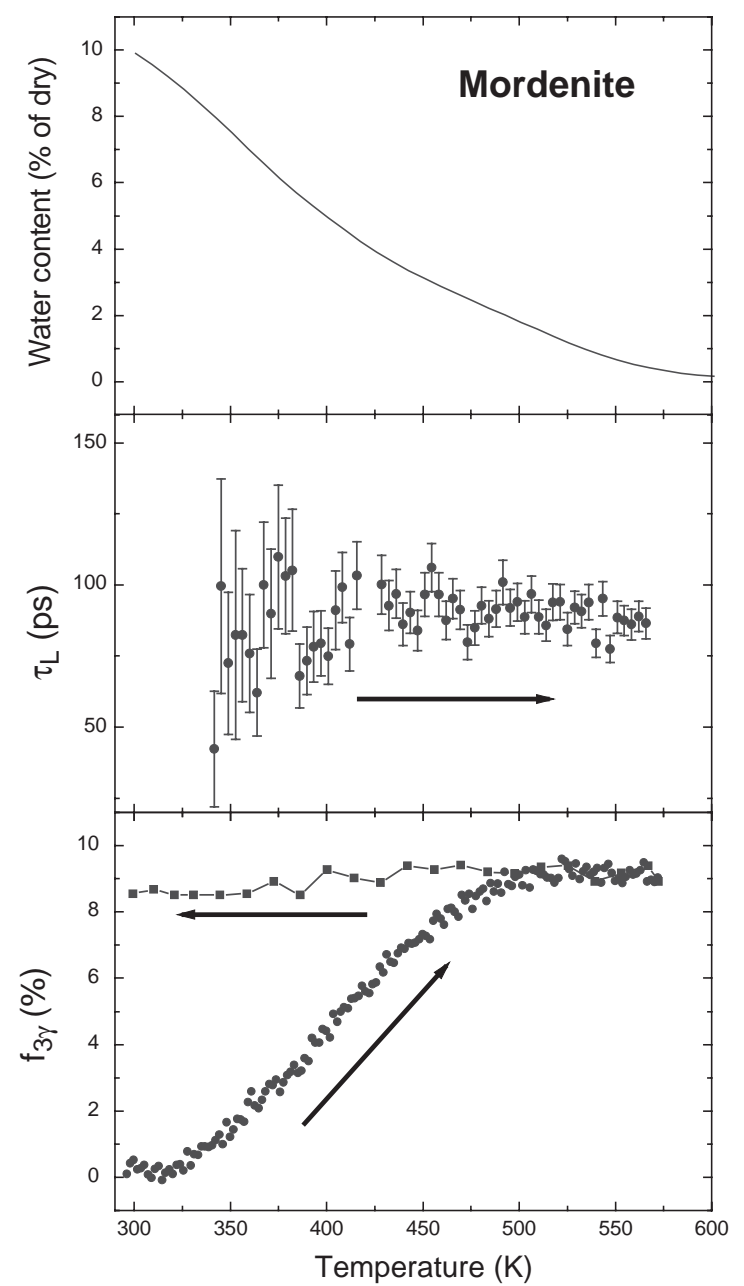

Fig. 2. Tg measurements and the variation of the long orthopositronium lifetime and its intensity upon water removal. The $f_{3 \gamma}, 3 \gamma$-fractions - determined using the results from the independent XDB measurements - are also given.

It is shown that for hydrophilic samples, it takes much effort to get rid of the water which may drastically change the PA parameter values and the relative occurrence of various annihilation channels in zeolites.

The corrections for the actual "birth population" relative intensities due to the shortening of lifetimes may strongly change the relative intensity values, including those of the short components as well. The non-linear detector response functions may introduce further distortions in the LT spectra, requesting further methodical investigations. Corrections of PA spectra due to changes in the occurrence of $3 \gamma / 2 \gamma$ annihilation and due to the energy dependence of the detector efficiency should be considered and carried out. It seems to be advisable to apply the magnetic quenching more extensively for a correct scaling of known relative changes in the unknown contribution of the 


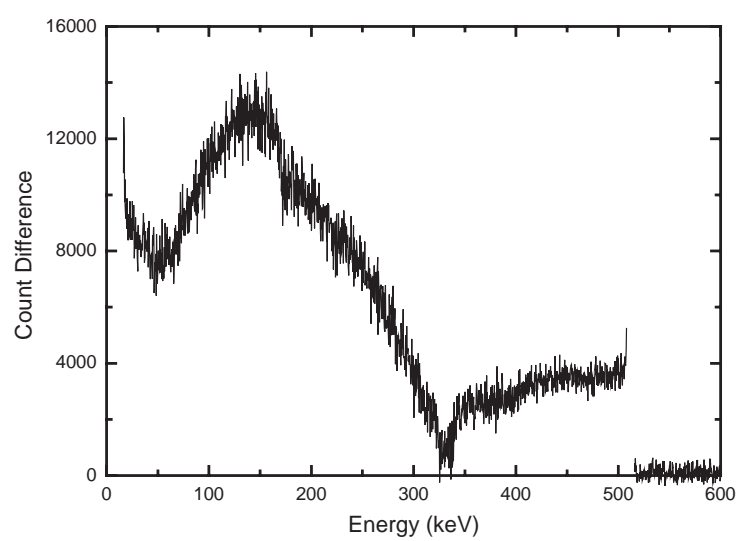

Fig. 3. Difference between the FED total energy distribution spectra for $\mathrm{Na}-\mathrm{Y}$ between water removed $(20 \%$ of long-living $o$-Ps is present) and water saturated (no $3 \gamma$-emitting $o$-Ps) states. The spectra are normalised to the same $1.28 \mathrm{MeV}$ peak area. The $511 \mathrm{keV}$ peak — having opposite sign — is not shown here.

$3 \gamma$-contribution. However, the values of the lifetimes are not influenced by those non-linear effects. A distribution of lifetime values in contrast to the discrete lifetime components models should also be considered in future studies.

A combined application of the various PAT methods is necessary to establish the proper intensity ratios and also for pinpointing the interplay between various annihilation channels coming to existence in the complex structural pattern of micro-and nanoporous media.

\section{Acknowledgements}

The present work was carried out partly in a cooperation program between the CNRS (France) and the Hungarian Academy of Sciences (HAS) and was also part of the French Program NOMADE. Partial supports by the OTKA Hungarian Research Fund, Grants T016506, T016294, T029215, T030327, T032029, T032249, F016293, W15585, W15722 and by the HAS Aid-In-Research Fund 98-116 2.2 are acknowledged. L.L. has benefited from a János Bolyai Research Fellowship, G.D. from a visiting fellowship within the European Commission FW5 Centre of Excellence project (contract No. ICA1-CT-2000-70029). Support through the Marie Curie-Fellowship for Zs.K. by the European Commission (contract HPMF-CT-200001038/MCFI-2000-01879) is recognised with appreciation.

Prof. T. Goworek, Dr. I. Billard and Dr. B. Molnár are thanked for advises and fruitful discussions, A. Bonnenfant for help in the sample preparation and in the PALS measurements.

\section{References}

Chen, Z.Q., Tang, Z., Wang, S.J., 1995. Surface properties of zeolite characterized by positron annihilation technique. Mater. Sci. Forum 175-178, 667.

Chezeau, J.M., Delmotte, L., Guth, J.L., Gabelica, Z., 1991. Influence of synthesis conditions and postsynthesis treatments on the nature and quantity of structural defects in highly siliceous MFI zeolites: a high resolution solid-state ${ }^{29}$ Si NMR study. Zeolites 11, 598.

Dauwe, Ch., 1992. On the evaluation of the fraction of orthopositronium in lifetime spectra. Mater. Sci. Forum 105-110, 1857.

Debowska, M., Abbé, J.Ch., Duplâtre, G., 1988. Positron annihilation studies in zeolites. Phys. Stat. Sol. B $146,91$.

Duplâtre, G., Kajcsos, Zs., Goworek, T., Varga, L., Liszkay, L., Billard, I., Lázár, K., 1996. Magnetic quenching effects on long-lived positronium states in zeosil. J. Radioanal. Nucl. Chem. 211 (1), 225.

Duplâtre, G., Kajcsos, Zs., Liszkay, L., Azenha, M.E., Paillaud, J.L., Soulard, M., Patarin, J.L., 2003. Positronium as a structural probe of MFI-type zeolites, submitted to Phys. Rev. B.

Eldrup, M., Lightbody, D., Sherwood, J.N., 1981. The temperature dependence of positron lifetimes in solid pivalic acid. Chem. Phys. 63, 51.

Goworek, T., Ciesielski, K., Jasinska, B., Wawryszczuk, J., 2000. Temperature variations of average $o$-ps lifetime in porous media. Radiat. Phys. Chem. 58, 719.

Guth, J.L., Kessler, H., Wey, R., 1986. New route to pentasiltype zeolites using a non alkaline medium in the presence of fluoride ions. In: Murakami, Y., et al. (Eds.), Proceedings of the Seventh International Zeol. Conference, Tokyo, Kodansha-Elsevier, Tokyo-Amsterdam, p. 121.

Hasegawa, M., Tsuchiya, Y., Kitayama, Y., Chiba, T., Saitoh, M., Yamaguchi, S., 1992. Positronium in zeolitic materialssepiolite. Mater. Sci. Forum 105-110, 257.

Ito, Y., Takano, T., Hasegawa, M., 1988. Positron annihilation is synthetic zeolites. Appl. Phys. A 45, 193.

Kajcsos, Zs., Liszkay, L., Varga, L., Lázár, K., Brauer, G., Dauwe, Ch., 1995. The mixing of $2 \gamma$-and $3 \gamma$-events in the data collection of positron annihilation experiments: microcrystalline powders and zeolites. Mater. Sci. Forum 175-178, 959.

Kajcsos, Zs., Duplâtre, G., Varga, L., Billard, I., Liszkay, L., Lohonyai, L., Caullet, P., Patarin, J., Lázár, K., 1997. Further results on long-lived positronium states in zeosil. Mater. Sci. Forum 255-257, 405.

Kajcsos, Zs., Duplâtre, G., Liszkay, L., Lázár, K., Lohonyai, L., Pál-Borbély, G., Beyer, H.K., Caullet, P., Patarin, J., 2001. Positrons and positronium in zeolites. Mater. Sci. Forum 363-365, 238-243.

Liszkay, L., Kajcsos, Zs., Duplâtre, G., Lázár, K., Pál-Borbély, G., Beyer, H.K., 2001. Positronium interactions in synthetic zeolites: effect of adsorbed water. Mater. Sci. Forum 363-365, 377-379.

Paulin, P., Ambrosino, G., 1966. Bound state of the positron in some finely divided oxides. C. R. Acad. Sci. Paris 263, 207.

Tao, S.J., 1972. Positronium annihilation in molecular substances. J. Chem. Phys. 56 (11), 5499-5510. 
Varga, L., Liszkay, L., Kajcsos, Zs., Lázár, K., Beyer, H.K., Onyestyák, G., Kótai, E., Lohonyai, L., 1996. Preliminary results of the positron annihilation in zeolites: peak shape and $3 \gamma$-decay. J. Radioanal. Nucl. Chem. 211 (1), 237.

Wang, S.J., Jean, Y.C., 1988. Positron annihilation spectroscopy of methane monolayers adsorbed on graphite. Phys. Rev. B 30, 4869.

Wang, S.J., Wang, B., Zhu, J., Wang, Z., Dai, Y.Q., He, C.Q., 2001. Positron and positronium in insulators. Mater. Sci. Forum 363-365, 377-379.

\section{Further reading}

Duplâtre, G., Kajcsos, Zs., Duplâtre, G., Kajcsos, Zs., Billard, I., Liszkay, L., Lázár, K., Lohonyai, L., Beyer, H.K.,
Caullet, P., Patarin, J., 1999. Free volumes in zeolites as seen by positrons. Stud. Surf. Sci. Catal. 125, $277-284$.

Huang, W.F., Hung, K.J., Huang, D.C., 2001. Application of ACAR spectroscopy to acidic catalysts. Mater. Sci. Forum 363-365, 263.

Ito, Y., Hirose, M., Tabata, T., Hasegawa, M., 1990. Positron annihilation in synthetic zeolites (II) magnetic quenching effect. Appl. Phys. A 50, 39.

Kajcsos, Zs., Dupltre, G., Liszkay, L., Billard, I., Bonnenfant, A., Azenha, E., Lázár, K., Pál-Borbély, G., Caullet, P., Patarin, J., Lohonyai, L., 2000. On the peculiarities of positron annihilation features in silicalite-1 and Y-zeolites. Radiat. Phys. Chem. 58, 709-714. 